\title{
Multilinguales
}

\section{Les difficultés de lecture en français langue étrangère chez les étudiants jordaniens}

Difficulties of reading in french foreign language in Jordan students

\section{Samer Hammouri}

\section{(2) OpenEdition}

\section{Journals}

\section{Édition électronique}

URL : http://journals.openedition.org/multilinguales/3807

DOI : $10.4000 /$ multilinguales.3807

ISSN : 2335-1853

Éditeur

Université Abderrahmane Mira - Bejaia

Référence électronique

Samer Hammouri, «Les difficultés de lecture en français langue étrangère chez les étudiants jordaniens », Multilinguales [En ligne], 10 | 2018, mis en ligne le 31 décembre 2018, consulté le 31 mai 2020. URL : http://journals.openedition.org/multilinguales/3807 ; DOI : https://doi.org/10.4000/ multilinguales.3807

Ce document a été généré automatiquement le 31 mai 2020.

\section{(c) (i) $(9)$}

Multilinguales est mise à disposition selon les termes de la Licence Creative Commons Attribution Pas d'Utilisation Commerciale - Pas de Modification 4.0 International 


\title{
Les difficultés de lecture en français langue étrangère chez les étudiants jordaniens
}

\author{
Difficulties of reading in french foreign language in Jordan students
}

Samer Hammouri

1 Apprendre une langue étrangère n'est pas seulement savoir communiquer, mais aussi perfectionner les quatre compétences. Dans l'histoire de l'enseignement des langues étrangères, l'expression écrite a été majoritairement privilégiée. La phonétique occupait une place souvent marginale dans les méthodes d'apprentissage. Cependant, depuis quelques années, nous assistons à un retour de l'enseignement de la prononciation en classe de langue et à l'introduction de la phonétique dans les méthodes de FLE.

2 L'un des problèmes d'apprentissage des langues étrangères est la lecture des textes dans cette langue et la maîtrise de la prononciation des mots.

Ce qui nous intéresse dans article est les problèmes phonétiques chez les apprenants

\section{Problématique}

3 La langue française est considérée comme la deuxième langue étrangère enseignée en Jordanie, après la langue anglaise. Lors de l'enseignement et de l'apprentissage de cette langue, les apprenants font face à plusieurs problèmes: grammaire, lecture, phonétique, etc. Dans les cours de lecture, nous avons constaté que les étudiants avaient beaucoup de mal à lire les textes à voix haute et qu'ils avaient des difficultés pour prononcer certains sons.

4 Le mot « lecture » peut renvoyer à deux réalités différentes :

- soit l'acte de lire : « la lecture à haute voix, la lecture courante et expressive» ;

- soit l'ouvrage lu : « avoir de bonnes, de mauvaises lectures ». 
Dans notre article nous nous intéressons à l'acte de lire et nous essayons de répondre aux questions suivantes :

Quels sont les problèmes phonétiques rencontrés par les étudiants jordaniens dans les universités publiques et privées en Jordanie?

Quelles sont les origines des difficultés de prononciation?

Comment pouvons-nous améliorer la prononciation chez les apprenants?

Quelle est la place des cours de phonétique dans les cursus universitaires?

6 Dans notre article, nous essaierons dans un premier temps de repérer les difficultés de prononciation lors de la lecture d'un texte écrit en français.

7 Notre article se base sur un questionnaire destiné aux étudiants jordaniens. Les apprenants concernés par cet article sont des les étudiants jordaniens de $2^{\text {ème }}, 3^{\text {ème }}$ et 4 ème année universitaire d'un seul établissement privé qui inscrit l'apprentissage du français dans son cursus universitaire. Ils sont âgés de 18 à 24 ans. Pour la constitution de notre corpus, nous avons sélectionné un échantillon de 60 étudiants de l'université du Zaytoonah pour répondre au questionnaire qui leur était destiné. Le nombre d'étudiants dans le département est de 200. Aussi nous avons choisi 20 étudiants de lire un texte pour relever les difficultés de prononciation lors de lecteur.

8 Les objectifs de ce questionnaire sont

Discerner les difficultés de prononciation de la langue française selon les étudiants.

Déterminer les ressources des ces problèmes phonétiques chez les étudiants.

9 Nous allons montrer l'importance de la conscience phonologique, et de la conscience phonémique dans le processus d'enseignement/apprentissage de la langue française dans les universités jordaniennes.

10 Ensuite, notre objectif sera d'analyser les nuances entre les phonèmes avec lesquels les apprenants ont des difficultés.

Nous essaierons de découvrir les causes et les origines de ces problèmes phonétiques en faisant une comparaison de sons entre les deux langues : l'arabe comme langue source et le français comme langue cible.

11 Enfin, nous proposerons des approches et des activités pédagogiques pour affronter/ surmonter ces types de problèmes.

\section{La conscience phonologique, et la conscience phonémique}

12 Pour bien parler une langue maternelle ou étrangère, il est nécessaire de connaître la prononciation des sons qui représente la base pour maîtriser cette langue.

13 Nous savons bien que le système phonique du français est composé de 16 voyelles, 17 consonnes et de 3 semi-consonnes. Pour les voyelles, on compte 12 orales et 4 nasales, parmi lesquelles $2 / 3$ sont articulées en antérieur. D'ailleurs, le système vocalique présente une forte proportion de voyelles labialisées. Pour les consonnes, on a les occlusives, les fricatives, les nasales, les orales, les sonores et les sourdes. Parmi lesquelles les sonores sont plus nombreuses que les sourdes. Ce qui fait que la langue française appartient aux langues à articulation antérieure, sonore et relativement douce. 
14 Nous pouvons dire que la conscience phonologique est un élément très important dans le développement des capacités en lecture. De nombreuses études abordent la conscience phonologique, comme celles de Bradley \& Bryant (1983), de Perfetti, l. Beck, Bell, Hughes. (1987), de Swank \& Catts (1994) etc.

Ces recherches ont affirmé que la conscience phonologique est l'habilité à identifier et à manipuler les mots dans une phrase et les parties d'un mot (les syllabes, les rimes et les phonèmes).

16 Les recherches de New and Cochran 2007 et Alfetcher 2006 ont montré que la conscience phonologique consiste à connaître les phonèmes/les sons/les mots. Cette habilité est en relation avec les processus de compréhension des mots du texte. Ces recherchent ont aussi affirmé que la connaissance de la conscience phonologique aide beaucoup les étudiants à mieux comprendre un texte.

17 Macellan (1999) a confirmé dans ses recherches que le perfectionnement de la lecture commence par la conscience phonologique.

18 A travers notre expérience en tant que professeur de français enseignant la lecture et la compréhension écrite, nous avons constaté que :

Les étudiants jordaniens ont beaucoup de difficultés pour prononcer les voyelles orales ou nasales : soit les lettres isolées comme [i], [o], [u], [e], soit les lettres liées comme «ou », « au », « eu ».

Tagliante (2006) a démontré que la confusion entre les voyelles nasales cause un grand problème pour les apprenants des langues étrangères. Ces problèmes sont en relation avec :

les sons au niveau de la labilité

les sons au niveau de la tension

les sons au niveau de l'acuité

Les étudiants arabophones en général et les étudiants jordaniens en particulier ont beaucoup de problèmes pour prononcer les graphèmes suivants :

19 Graphème an, c'est celui de l'ensemble des participes présents de tous les verbes français : aimant (amare), lisant (legere), délayant (delere), etc. Nous retrouvons "an" dans quelques adjectifs et noms terminés en -mant (issus des verbes en - mer, amant, ou de leurs formes lexicalisées, charmant, calmant), lesquels s'opposent nettement aux finales en -ment.

Graphème en, on peut se rattacher aux séries suivantes : - Á l'initiale, au préfixe entre (inter). - Á la finale, essentiellement au suffixe -ment (latin -mente).

21 Graphème in (im), la voyelle i en dérivation grammaticale ou lexicale, exemples : fin, burin, destin, etc., la dérivation se fait parfois en -igne, exemple : maligne, malignité, etc.

22 Graphème ain (aim), présente les mêmes alternances et se trouve dans les mêmes séries paradigmatiques. - Noms et adjectifs féminins en - : sain, vain, etc. - Les dérivés des mots en -aim sont en ai $+m$ : faim, essaim, etc.

23 Graphème ein, présente les mêmes alternances que ei, assez rare d'ailleurs : - Noms et adjectifs en -eine : plein, serein.

24 Nous constatons que les consonnes sont définies de façon plus précise que les voyelles, du fait que les consonnes sont mieux localisées, grâce à l'utilisation de plus de traits de nasalité et de labilité. En revanche, pour définir la localisation des voyelles, on utilise 
les notions suivantes : aperture, antériorité, postériorité, nasalité « caractérise les sons vocaux qui sont produits sans lever le voile du palais, c'est-à-dire on laisse l'air passer par le nez. ${ }^{1}$ " plusieurs approches :

L'approche Orton-Gillingham :

Cette approche multi sensorielle amène les quatre types de connexions neuronales (visuelles, auditives, tactiles et kinesthésiques) à être engagées lors de l'apprentissage de la lecture.

L'approche Orton-Gillingham pour l'acquisition de la lecture qui a été développée dans les années 1930 est toujours reconnue aujourd'hui comme étant la méthode la plus efficace de rééducation pour la dyslexie.

L'approche Ferland :

Elle n'est pas tout à fait différente de l'approche Orton-Gillingham, mais dans cette approche, les étudiants doivent utiliser les trois sens pour développer leur capacité de lecteur. Pour lire un texte il faut utiliser nos sens; voir le mot (la vue), ensuite, prononcer le mot (l'ouie), puis, suivre le mot avec les doigts (le toucher).

L'approche neurologique :

L'enseignant lit à haute voix et les apprenants répètent, ou l'ensemble de la classe, l'enseignant et les étudiants, lit en même temps, mais l'enseignant doit parler un peu plus fort. Dans ce cas-là, l'enseignant doit choisir des textes courts et variés.

\section{Méthodes de correction de la prononciation}

Autrefois, les méthodes de l'enseignement du français accordent beaucoup d'importance à la grammaire et à la traduction. La phonétique a été exclue des considérations des pédagogues.

Avec l'apparition de l'alphabet phonétique international (1880-1890), la phonétique a sérieusement commencé à avoir sa place dans l'enseignement des langues 
(Guimbretière, 1994). C'est la méthodologie directe. Ainsi la maîtrise de la prononciation a-t-il été considérée comme un préalable à toute présentation du code écrit. Cette méthode propose des exercices comme la dictée phonétique, la transcription phonétique aux enseignants, aux apprenants. Cette méthodologie est conçue comme la première étape dans l'acquisition des compétences phonétiques.

\section{La méthode articulatoire}

Dans cette méthode, l'enseignant fait la description claire et simple des mouvements nécessaires de l'appareil phonatoire pour la réalisation des différents phonèmes. Ensuite, il doit visualiser les diverses positions des organes phonatoires. Par exemple, il montre l'arrondissement des lèvres pour la prononciation de certaines voyelles nasale/ orales; ou comment faire vibrer les cordes vocales pour certaines consonnes; ou comment mettre les doigts sur le nez pour montrer les sons des nasales.

Le rôle de l'apprenant dans cette méthode est de savoir décomposer les sons en un nombre de gestes articulatoires nécessaires pour sa production. Il doit pratiquer et mémoriser ces gestes articulatoires et acquérir des automatismes de prononciation parfaite des sons.

33 L'objectif principal de la méthode articulatoire est l'acquisition consciente de la prononciation. Selon R. Renard (1971), cette méthode néglige le facteur auditif, les phénomènes combinatoires et de compensation ainsi que les éléments prosodiques, elle surestime l'aspect conscient de la phonation en ignorant le caractère global du comportement langagier et inhibe également la spontanéité de l'expression (Renard, 1971).

\section{La méthode des oppositions phonologiques}

La méthode des oppositions phonologiques, appelée aussi la méthode des traits distinctifs, est basée sur les traits distinctifs selon lesquels les phonèmes ont été classés. Cette méthodologie s'appuie sur la mémorisation des phonèmes à travers l'opposition des paires minimales comme [s]-[z].

Nous pouvons proposer dans cette méthode des exercices sur l'opposition entre deux ou plusieurs phonèmes. Les apprenants doivent écouter et répéter des listes de mots où le changement d'un seul son apporte un changement de sens. Par exemple : "le / les"; "basse / base" ; "bon / vont", etc.

Les apprenants peuvent faire appel à des outils et à des technologies et des matériels audio ou vidéo pour la correction de la prononciation, comme le magnétophone, le laboratoire de langues ou l'ordinateur. Beaucoup de manuels et de cours de prononciation proposent des activités d'opposition. Cette méthode offre la possibilité de travailler en autonomie, en classe, dans le laboratoire ou en salle multimédia. Les défauts cette méthode sont qu'elle privilégie l'élément isolé, il n'existe pas de continuum sonore. Par conséquent, il n'y a aucune considération pour la prosodie.

\section{La méthode verbo-tonale}

La méthode verbo-tonale a été créée dans les années 1950-60 par Petar Guberina.

Guberina a donné beaucoup d'importance au rythme et à l'intonation qui :

constituent un moyen puissant de reconnaissance des sonorités langagières ; 
relient entre eux les sons successifs : voyelles et consonnes ;

interviennent directement sur la réalisation des segments paroliers : tout son est obligatoirement inséré à l'intérieur d'une structure rythmico-intonative et en subit forcément l'influence ;

suffisent parfois, quand judicieusement utilisés, à éradiquer une erreur donnée portant sur une voyelle ou une consonne.

Il s'ensuit que le praticien de la méthode verbo-tonale corrigeant un son défectueux doit toujours envisager l'influence exercée par le rythme et l'intonation.

La méthodologie verbo-tonale s'oppose aux approches articulatoires, qui décrivent les positions des organes phonatoires. C'est une méthode SGAV (Structuro Globale Audio Visuelle) renommée SG \&VT (et Verbo Tonal).

Cette méthode part d'un diagnostic des erreurs de prononciation de l'apprenant, l'enseignant fait répéter par les apprenants des phrases courtes et simples, en appliquant divers procédés correctifs : déformer les sons, le rythme et la mélodie d'une phrase de la langue cible, l'apprenant entend/prononce et acquiert les sons inconnus.

\section{Origine des problèmes phonétiques chez les étudiants jordaniens}

2 Dans le domaine de la phonétique, chaque langue a ses caractéristiques spéciales. Les différentes caractéristiques des langues présupposent un système phonétique différent, ce qui amène à la difficulté de la perception et de la production de certains éléments lorsqu'on apprend une langue étrangère.

Nous pensons que l'influence de la langue maternelle arabe est importante, c'est pourquoi nous prononçons mal les voyelles car ces voyelles n'existent ni dans la langue maternelle des étudiants, qui est l'arabe, ni dans la première langue étrangère acquise (anglais). Troubetzkoy (1986) a abordé la notion de crible phonologique ou système d'écoute contrôlé par le système phonologique de la langue maternelle, qui perturbe l'identification et l'articulation des sons d'une langue étrangère.

Nous pouvons ajouter aussi que, les apprenants vont avoir tendance à rapprocher les sons de la langue cible du système phonologique de leur langue maternelle. C'est pourquoi les erreurs que les apprenants commettent varient toujours selon leurs origines.

5 La deuxième raison pour comprendre les difficultés de prononciation des voyelles rencontrées par les apprenants est l'insuffisance des cours de phonétique dès la première année ou dans certains cas l'absence totale de cours de phonétique dans les cursus des universités jordaniennes.

Nous pouvons ajouter aussi le manque de pratique et d'entraînement sur les voyelles nasales dans la classe de FLE.

Dans les matières d'initiation (débutants ou première année), l'enseignant n'a pas assez de temps pour expliquer ou pratiquer les nuances entre les voyelles, etc.

Enfin, le manque d'écoute des émissions/chansons en français et des natifs pose problème, car pratiquer ces activités aide beaucoup les étudiants à développer leurs compétences de la compréhension orale et de la compréhension écrite. 


\section{La place de la phonétique dans les cursus des universités jordaniennes} de phonétique) en essayant de répertorier l'importance et la présence des cours de phonétique destinés aux étudiants de la première année jusqu'à la fin des études.

L'Université de Jordanie propose aux étudiants trois cours :

La compréhension orale

La compréhension orale et écrite (français 1)

La compréhension orale et écrite (français 2)

L'Université Yarmouk, Département des langues modernes

La production orale (1)

La production orale (1)

La production orale (2)

La compréhension écrite

La compréhension orale

La phonétique (optionnelle) : ce cours - LM 249 : Introduction à la phonétique française (3 heures) a pour objectif d'initier les apprenants à quelques notions phonétiques de base. Cela consiste à expliquer la relation entre le son et les lettres de la langue française. Le pôle théorique présente toutes les règles de classification des sons et leur prononciation exacte.

Le département des langues européennes à l'université du Mutah propose les cours suivants :

La compréhension et la production orale

Phonétique

Lecture et écrit en français

La phonétique et la compréhension orale

L'Université Al-Zaytonah, département de français et d'anglais et leur littérature :

Entrainements phonétiques et langagiers 1

Entrainements phonétiques et langagiers 2

Nous pouvons remarquer qu'il y a juste deux universités qui donnent des cours à proprement parler de phonétique, l'université du Yarmouk et l'université du Mutah, ce fait peut être expliqué par le manque d'enseignants spécialisés en phonétique.

\section{Proposition}

Nous voudrions conclure ce travail en donnant quelques recommandations pour améliorer l'habilité phonétique chez les apprenants :

il est conseillé de consacrer assez de temps pour enseigner explicitement les voyelles en donnant des exercices variés oraux, par exemple, écouter des chansons et voir des films français dans la classe ;

utiliser les chansons dans la classe est un moyen efficace aussi pour développer la compétence de compréhension orale ;

nous proposons aux enseignants de faire des activités qui peuvent aider les apprenants à prononcer et distinguer les voyelles telles que : - Exercice à trous ; 
l'enseignant peut aussi utiliser la gestualité dans la classe lorsqu'il donne des explications sur la prononciation et la phonétique. Selon l'ouvrage de Cormanski (2005), pour apprendre la phonétique et à prononcer correctement, le corps de l'apprenant est le premier constituant authentique de la classe et apparaît comme un moyen qui véhicule du sens ;

il peut illustrer la différence entre les deux phonèmes [p] et [b], en utilisant une feuille de papier : il prononce le [p] devant les apprenants en montrant que le papier a bougé, et prononce le [b] en montrant qu'il ne bouge pas, ensuite il demande aux apprenants de sortir une feuille et de faire pareil ;

les enseignants doivent être attentifs lorsqu'ils peuvent demander ou répondre car la prononciation des voyelles est très difficile ;

L'utilisation de Français natifs est nécessaire comme un bon modèle.

Créer un laboratoire disponible tout au long de la journée pour donner l'occasion aux apprenants d'utiliser ce laboratoire pendant les pauses.

D'après le graphique en cascade de York Region District School Board « Comprendre les troubles d'apprentissage : incidence des domaines de traitement sur l'apprentissage », les élèves ayant des troubles d'apprentissage ou des difficultés d'apprentissage qui atteignent la conscience phonologique pourraient démontrer des difficultés pour :

reconnaître des mots qui riment ;

trouver des mots qui riment ;

découper un mot en unités (segmentation en syllabes);

trouver le nombre de mots dans une phrase en tapant dans les mains ;

segmenter les sons dans un mot (p.ex. [o-r-a-g-e]);

combiner des sons pour produire des mots ;

se remémorer des sons dans des mots parlés et écrits;

relier sa conscience des sons à sa connaissance des symboles qui représentent ces

sons. (2014, p. 3).

Pour conclure, il faudrait noter que la conscience phonétique est un élément constituant de l'enseignement/apprentissage de la langue maternelle ou étrangère.

Pour perfectionner une langue étrangère, il faut prendre en compte l'importance des sons dans la langue cible. Cela aide les étudiants à développer leurs compétences de compréhension écrite et de compréhension orale.

$\mathrm{Au}$ niveau du système universitaire, nous pensons que la phonétique doit être intégrée dans l'étude de la langue comme matière obligatoire des la première année. Ces cours doivent s'appuyer sur les éléments de base de la phonétique et sur la correction phonétique et bien sûr aider les apprenants à différencier les voyelles.

La présence d'un laboratoire à la disponibilité des étudiants, dans lequel les apprenants peuvent pratiquer la langue française, est un moyen d'apprentissage efficace.

La première catégorie contient les problèmes qui proviennent de l'influence de la langue arabe ou d'autres langues étrangères apprises par les apprenants. 


\section{BIBLIOGRAPHIE}

La deuxième catégorie comporte les problèmes qui proviennent de la complexité phonétique propre à la langue française.

BRADLEY, L. et BRYANT, P.E., "Categorizing sound and learning to read" : A causal connection, in. Nature, $n^{\circ} 303,419-421,1983$.

GUIMBRETIÈRE, E., Phonétique et enseignement de l'oral, Didier/HATIER, Paris, 1994.

RENARD, R., Introduction à la méthode verbo-tonale de correction phonétique, Didier, Paris, 1971.

PERFETTI, C. A, Beck, I., Bell, L. C et Hughes, C., "Phonemic knowledge and learning to read are reciprocal " : A longitudinal study of first grade children, in. Merrill-Palmer Quarterly, vol. 33, No. 3, 1987, pp. 283-319.

SWANK, L. ; CATTS, H.W. "Phonological awareness and written word decoding", in Language, speech and hearing in schools, 25, 1994, 9-14.

TAGLIANTE Christine, La classe de langue, CLE international, Paris, 2006.

TROUBETZKOY, N.S., Principes de Phonologie, Klincksieck, Paris, 1986.

\section{NOTES}

1. 13 ZRARI, Siham, module de phonétique, université Mohammed kheider, Biskra, Algérie ,2011 / 2012 »

\section{RÉSUMÉS}

Dans cet article, nous essayerons d'expliquer les difficultés rencontrées par les étudiants qui apprennent le français aux universités jordaniennes. Nous supposons que le problème principal réside dans la difficulté de distinguer et de prononcer les voyelles nasales et orales. Nous proposerons des activités pour la correction phonétique afin de résoudre ces problèmes. À la fin de l'article, nous suggérons certaines recommandations et conseils pour résoudre ces problèmes phonétiques.

In this article we try to explain the phonetic problem with students who learn French language in Jordan's universities. We found the main problem is to differentiate between vowels and letters. We propose in this article more activities to teachers that help them to solve this problem. We try also to understand the origin of this problem. At the end of this article, we suggest some recommendations for teachers, students and university. 
INDEX

Keywords : pronunciation, French, vowels, prosodies, phonetic, phonology, phonetic correction Mots-clés : prononciation, apprentissage, français, voyelle, consonne, prosodie, phonétique, phonologie, correction

\section{AUTEUR}

\section{SAMER HAMMOURI}

Université Al- Zaytoonah, Amman - Jordanie 